\title{
Représentation topologique $(p, T)$ du trimorphisme du ferrocène : métastabilité intrinsèque de la phase triclinique
}

\author{
S. Toscani, P. De Oliveira ${ }^{1}$ et R. Céolin \\ Laboratoire de Chimie Physique, Faculté de Pharmacie, 4 avenue de l'Observatoire, \\ 75006 Paris, France \\ ${ }^{1}$ Laboratoire de Biophysique, Muséum National d'Histoire Naturelle, 43 rue Cuvier, \\ 75005 Paris, France
}

\begin{abstract}
Structural and thermodynamic data reported in literature about the three crystalline phases of ferrocene are used to build up a topological representation of its $(\mathrm{p}, \mathrm{T})$ phase diagram. Two phases (orthorhombic and monoclinic) exhibit stable phase regions whose temperature ranges increase with increasing pressure. The triclinic phase is found to be metastable whichever temperature and pressure. In the ( $\mathrm{p}, \mathrm{T})$ diagram, it is not associated with any state of lowest energy although it transforms into the monoclinic phase according to a transition which is reversible. It is shown that this occurs in the phase region where the orthorhombic phase is the stable phase.
\end{abstract}

\section{Introduction}

Grâce à l'ensemble des données structurales et thermodynamiques trouvées dans la littérature, le trimorphisme du ferrocène est présenté comme un autre exemple de détermination, en fonction de la température et de la pression, de la hiérarchie de stabilité des phases cristallines d'une espèce chimique. Cette hiérarchie résulte de l'application de la méthode de construction des diagrammes de phases (p,T), fondée sur la localisation topologique et l'établissement des degrés de stabilité des points triples.

La bibliographie sur ce composé «sandwich» montre que les structures cristallines des trois phases solides connues (monoclinique $\mathrm{M}$, triclinique $\mathrm{T}$, et orthorhombique $\mathrm{O}$ ) ont toutes été résolues [1-4]. Sur le plan thermodynamique, il est bien connu depuis le travail de Edwards et al [5] que, par refroidissement au dessous de $200 \mathrm{~K}$, la phase $\mathrm{M}$, stable à l'ambiante, se transforme dans la phase T à $164 \mathrm{~K}$. Toutefois, Ogasahara et al [6] découvrirent en 1979 l'existence d'une nouvelle phase solide à basse température, de structure orthorhombique, ayant une chaleur spécifique plus faible que celle de la phase $\mathrm{T}$. Bien que la transition $M \rightarrow T$ observée dans ce sens au refroidissement soit toujours observée en sens inverse à l'échauffement, ces auteurs concluent que la phase $\mathrm{O}$ est stable à des températures inférieures à $242 \mathrm{~K}$, à savoir la température de la transition $\mathrm{O} \rightarrow \mathrm{M}$ qu'ils observent à l'échauffement.

Par conséquent, la transition T-M à $164 \mathrm{~K}$ aurait lieu entre phases métastables. La construction du diagramme de phases $(p, T)$ du ferrocène a donc été entreprise dans le but de :

- confirmer les conclusions de Ogasahara et al sur la métastabilité de la transition réversible T-M,

- établir si la phase $T$ est intrinsèquement métastable, c.-à-d. non associable à un domaine de stabilité maximale dans le diagramme $(\mathrm{p}, \mathrm{T})$, ou si, au contraire, elle montre un comportement énantiotrope à haute pression,

- établir si les domaines de stabilité des phases $\mathrm{O}$ et $\mathrm{M}$ sont limités ou non en pression. 


\section{Construction du diagramme de phases}

Les coordonnées de tous les points triples comportant la phase vapeur $v$ (ils se situent à de pressions proches de $0 \mathrm{MPa}$ ) ont d'abord été calculées. En assimilant les températures des transitions T-M et O-M à celles des points triples T-M- $v$ et O-M- $v$ et la température de fusion de la phase $\mathrm{M}$ à celle du point triple M- $l$ $v$, il restait à déterminer les températures de fusion des phases $O$ et $T$ et celle de la transition $O-T$, de manière à définir les coordonnées des points triples $\mathrm{O}-l-v, \mathrm{~T}-l-v$ et $\mathrm{O}-\mathrm{T}-v$. Dans ce but, les équations expérimentales de l'équilibre de sublimation de la phase $M$ [7] et de l'équilibre liquide-vapeur [8] sont utilisées. L'intersection de ces deux courbes restitue correctement la température de fusion de la phase $M(448 \mathrm{~K})$.

Ensuite, en tenant compte que, dans l'équation $\ln \mathrm{p}=\mathrm{A}-\mathrm{B} / \mathrm{T}$ appliquée à un équilibre de sublimation, la constante $\mathrm{B}$ est proportionnelle à l'enthalpie de sublimation, les valeurs des coefficients $\mathrm{B}$ et les équations des équilibres de sublimation ont été calculées pour les phases $O$ et $T$. Leurs enthalpies de sublimation ont été estimées grâce au premier principe de la thermodynamique $\left(\Delta_{\mathrm{O} \rightarrow \mathrm{v}} \mathrm{H}=\Delta_{\mathrm{O} \rightarrow \mathrm{M}} \mathrm{H}+\Delta_{\mathrm{M} \rightarrow \mathrm{v}} \mathrm{H}\right.$ et $\left.\Delta_{\mathrm{T} \rightarrow \mathrm{V}} \mathrm{H}=\Delta_{\mathrm{T} \rightarrow \mathrm{M}} \mathrm{H}+\Delta_{\mathrm{M} \rightarrow \mathrm{V}} \mathrm{H}\right)$. Les coefficients $\mathrm{A}$ ont été obtenus en égalisant les tensions de vapeur des phases $\mathrm{O}$ et $\mathrm{T}$ à celles de la phase $\mathrm{M}$ aux températures des transitions $\mathrm{O}-\mathrm{M}$ et $\mathrm{T}-\mathrm{M}$. Enfin, les températures des points triples de fusion $\mathrm{O}-l-\mathrm{v}$ et $\mathrm{T}-l-\mathrm{v}$ ont été calculées en résolvant les systèmes entre l'équation de la courbe de vaporisation du liquide et celles des équilibres de sublimation des deux solides. Il s'ensuit que la fusion de la phase $\mathrm{M}$ est stable car elle est située sur le diagramme à la température la plus élevée (445 $\mathrm{K}$ ), que celle de la phase $\mathrm{T}(422,2 \mathrm{~K})$ est métastable et que celle de la phase $\mathrm{O}(399,5 \mathrm{~K})$ est supermétastable (Figure 1). Par ailleurs, la métastabilité de la transition T-M est confirmée car le point triple $\mathbf{M}-\mathrm{T}-\nu$ est métastable. En effet, il est situé sur le prolongement métastable (vers les basses températures) de la portion stable de l'équilibre M-v (ligne 3, Fig. 1), limitée par les deux points triples stables $\mathbf{M}-l-v$ et $\mathrm{O}-\mathrm{M}-v$. Le point triple O-T- $v(275 \mathrm{~K})$ est un autre point triple métastable car il est localisé sur un prolongement métastable de l'équilibre O-v (ligne 1, Fig. 1), stable à basse température ( $\mathrm{T} \leq 242 \mathrm{~K}$ )

La position du point triple $O-T-M$ sur le diagramme se trouve à l'intersection des droites des équilibres O-M et T-M. Afin d'en déterminer les équations, leurs pentes $\mathrm{dp} / \mathrm{dT}$ ont été calculées par l'équation de Clapeyron. Les variations d'enthalpie étant celles des transitions solide-solide respectives, les variations de volume ont été estimées aux températures correspondantes grâce aux équations donnant les volumes spécifiques des phase $\mathrm{M}$, $\mathrm{T}$ et $\mathrm{O}$ en fonction de la température. Le point triple O-T-M apparait alors comme situé à pression négative. Ce résultat, excluant que la phase $\mathrm{T}$ puisse posséder un domaine de stabilité à haute pression, montre qu'il s' agit donc d'une phase intrinsèquement métastable.

Le fait que des points triples soient localisés à des pressions négatives est dépourvu de sens physique précis. Il découle du fait que des équations linéaires sont choisies pour représenter les équilibres $S_{i}-S_{j}$ et $S_{i}-l$. Néanmoins, ce choix se révèle très judicieux pour la localisation et la représentation topologique des points triples sur le diagramme de phases.

Les trois derniers points triples, O-M- $l$, O-T- $l$ et M-T- $l$, ont également été localisés à des pressions négatives. Ceci entraîne que les équilibres de fusion $\mathrm{S}_{\mathrm{i}}-l$ des trois phases divergent en température quand la pression augmente et que donc les domaines de stabilité des phases $\mathrm{O}$ et $\mathrm{M}$ ne sont pas limités en pression. Autrement dit, ces deux phases présentent un comportement énantiotrope généralisé. Il faut souligner que cette divergence des équilibres de fusion, déduite de la localisation topologique des points triples, est confirmée par le calcul de leurs pentes, effectué en utilisant les variations d'enthalpie et de volume mesurées ou calculées à partir des données expérimentales.

\section{Conclusion}

Le diagramme $(\mathrm{p}, \mathrm{T})$ du ferrocène montre que, des trois phases solides connues, seules les variétés orthorhombique et monoclinique possèdent des domaines d'existence qui correspondent à des états de moindre énergie. La première est stable à basse température (pour $\mathrm{T} \leq 242 \mathrm{~K}$ à pression ordinaire), la deuxième entre la température de la transition $\mathrm{O}-\mathrm{M}$ et celle de la fusion de la phase $\mathrm{M}$. Leurs domaines de stabilité s'élargissent en température lorsque la pression augmente, ce qui est caractéristique d'un comportement énantiotrope généralisé. En revanche, la phase triclinique est intrinsèquement métastable, car elle ne possède pas de domaine d'existence correspondant à des conditions $p$ et $T$ de moindre énergie du 
système. Les conclusions de Ogasahara portant sur le caractère métastable de la transition reversible T-M à $164 \mathrm{~K}$ sont donc confirmées : elle a lieu dans le domaine de stabilité de la phase orthorhombique.

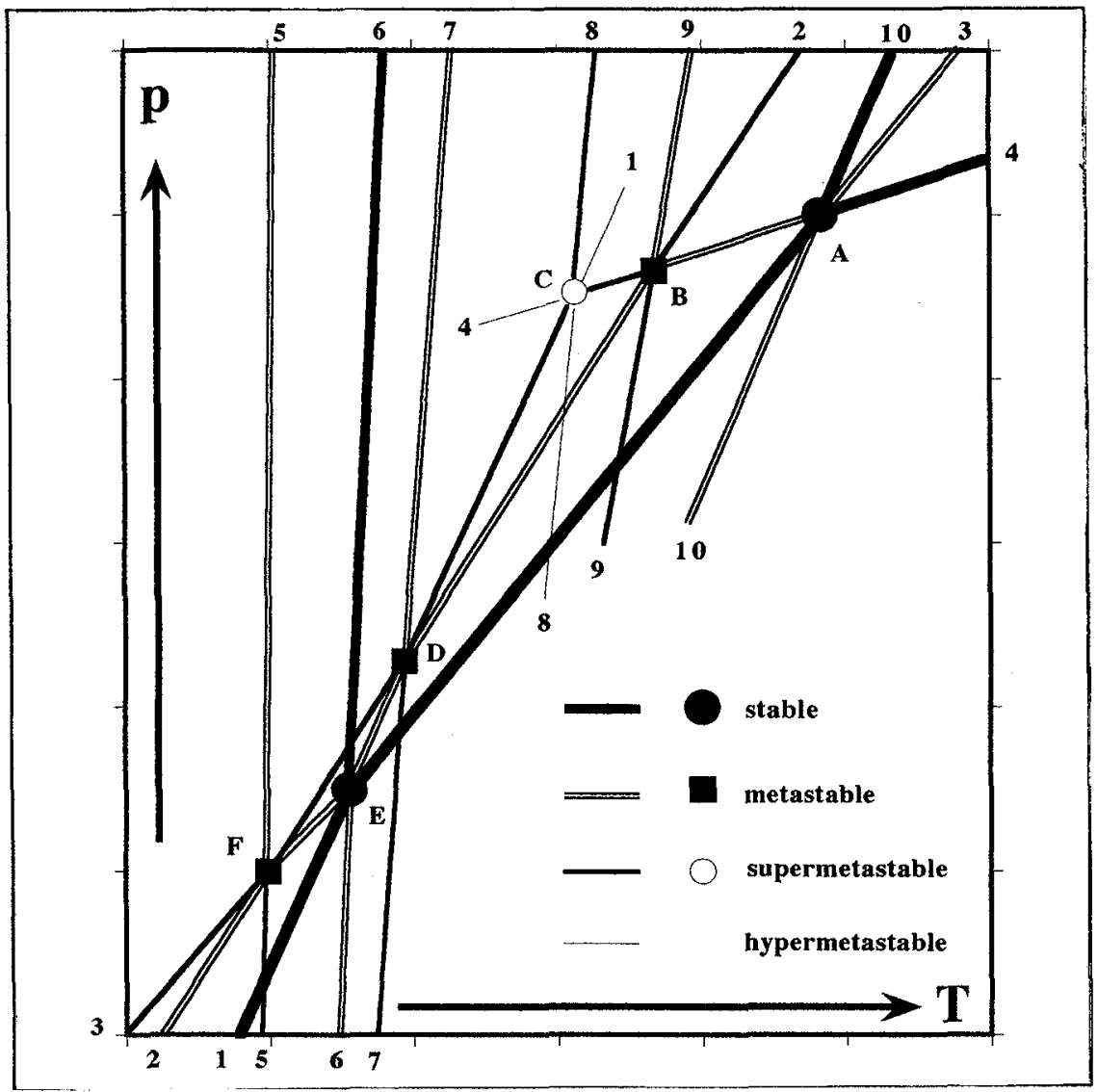

FIGURE 1: Représentation topologique du diagramme $p, T$ du ferrocène.

Équilibres diphasés. $1=\mathrm{O}+v, 2=\mathrm{T}+v, 3=\mathrm{M}+v, 4=l+v, 5=\mathrm{T}+\mathrm{M}, 6=\mathrm{O}+\mathrm{M}, 7=\mathrm{O}+\mathrm{T}, 8=\mathrm{O}+l, 9=\mathrm{T}+l$, $10=\mathrm{M}+l$. Points triples: $\mathrm{A}=\mathrm{M}-l-v, \mathrm{~B}=\mathrm{T}-l-v, \mathrm{C}=\mathrm{O}-l-v, \mathrm{D}=\mathrm{O}-\mathrm{T}-v, \mathrm{E}=\mathrm{O}-\mathrm{M}-v, \mathrm{~F}=\mathrm{T}-\mathrm{M}-v$.

\section{Références}

[1] Seiler P. et Dunitz J. D., Acta Cryst. B35 (1979), 1068-1074.

[2] Takusagawa F. et Koetzle F., Acta Cryst. B35 (1979), 1074-1081.

[3] Seiler P. et Dunitz J. D., Acta Cryst. B35 (1979), 2020-2032.

[4] Seiler P. et Dunitz J. D., Acta Cryst. B38 (1982), 1741-1745.

[5] Edwards J. W., Kington G. L. et Mason R., Trans. Faraday Soc. 56 (1960), 660-667.

[6] Ogasahara K., Sorai M. et Suga H., Chem. Phys. Lett. 68 (1979), 457-460.

[7] J. W. Hiby et M. Pahl, Z. Naturforsch. 7a (1952), 542-553.

[8] Scherer F., Fischer E. O. et Grubert H., Chem. Ber. 92 (1959), 2302-2309. 